\title{
Clinical heterogeneity among a three-generation Japanese family with D18N TREX1 mutation for Aicardi-Goutières syndrome / familial chilblain lupus
}

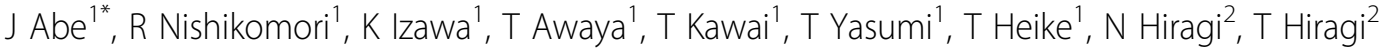 \\ From 18th Pediatric Rheumatology European Society (PReS) Congress \\ Bruges, Belgium. 14-18 September 2011
}

\section{Background}

Aicardi-Goutières syndrome (AGS) is a genetic disease, characterized by encephalopathy with cerebral calcification, white matter abnormalities, cerebral atrophy, elevated interferon-alpha in the cerebrospinal fluid and chilblain. Most of AGS patients have severe neurological findings including developmental delay. Five genes, namely TREX1, RNASEH2B, RNASEH2C, RNASEH2A, SAMHD1 have been reported to be responsible for AGS. Most cases of AGS are inherited as autosomal recessive manner, although autosomal dominant AGS is rarely reported and its clinical manifestations are largely unknown.

\section{Aim}

We found a three-generation Japanese family whose members shared severe chilblain in an autosomal dominant manner. We tried to identify the responsible gene and investigated genotype-phenotype correlation within the family.

\section{Methods}

We performed sequencing of the genes responsible for AGS and obtained clinical information from the family.

\section{Results}

The proband had severe chilblain, mental retardation, cerebral calcification, and elevated serum interferon-alpha. We identified a heterozygous TREX1 mutation, D18N $(52 \mathrm{G}>\mathrm{A})$ and diagnosed her as AGS. Her mother had the same D18N mutation, although she lacked neurological impairments and cerebral calcification was comparable to the age-matched controls. We diagnosed her mother as familial chilblain lupus (FCL). The proband's nephew also shared the same D18N mutation with neurological abnormalities including mental retardation and epilepsy. However, he lacked cerebral calcification and chilblain. These data indicated that the same TREX1 mutation D18N caused AGS, FCL, and the neurological disorder without brain calcification in the same family.

\section{Conclusion}

It should be alerted that autosomal dominant TREX1 mutation $\mathrm{D} 18 \mathrm{~N}$ has clinical variation when performing genetic test for AGS/FCL.

\section{Author details}

'Department of Pediatrics, Kyoto University Graduate School of Medicine, Kyoto, Japan. ${ }^{2}$ Department of Pediatrics, Municipal Tsuruga Hospital, Tsuruga, Japan.

Published: 14 September 2011

doi:10.1186/1546-0096-9-S1-P276

Cite this article as: Abe et al: Clinical heterogeneity among a threegeneration Japanese family with D18N TREX1 mutation for AicardiGoutières syndrome / familial chilblain lupus. Pediatric Rheumatology 20119 (Suppl 1):P276.

\footnotetext{
* Correspondence: junyaabe@kuhp.kyoto-u.ac.jp

'Department of Pediatrics, Kyoto University Graduate School of Medicine, Kyoto, Japan

Full list of author information is available at the end of the article
}

(c) 2011 Abe et al; licensee BioMed Central Ltd. This is an open access article distributed under the terms of the Creative Commons 